\section{Risk management in clinical practice. Part 7. Dento-legal aspects of orthodontic practice}

E. Mizrahi ${ }^{1}$

VERIFIABLE CPD PAPER
IN BRIEF

- One of the risks for patients undergoing orthodontic treatment is the development of carious lesions around appliances.

- Careful clinical examination is required and specific attention paid to TMJ signs and symptoms, periodontal tissues and previous trauma to teeth.

- The clinician needs to be aware of the possible risks associated with orthodontic treatment and advise the patient or parent accordingly.

Orthodontic treatment, like any aspect of general dentistry, exposes the clinician to the risk of malpractice and litigation. While for orthodontists this issue has not been a major concern over the last century, risk management has, over the last decade, become a significant issue in orthodontic practice. The patient-orthodontist contact may be classified into pre-treatment, active treatment and post-treatment periods. Risk management issues pertinent to each time period are discussed in this article with the intention of increasing the clinician's awareness of potential problems. Armed with this knowledge the overriding message for the clinician is to practise orthodontics with the philosophy of prevention and avoidance. To the best of your ability, avoid giving patients a reason to institute legal proceedings.

\section{INTRODUCTION}

In orthodontics, the risk of harm to the patient is considerably limited compared to disciplines that involve surgery and other invasive therapies. However, the extent of malpractice has, over the last

\section{RISK MANAGEMENT IN CLINICAL PRACTICE \\ 1. Introduction \\ 2. Getting to 'yes' - the matter of consent \\ 3. Crowns and bridges \\ 4. Endodontics \\ 5. Ethical considerations for dental enhancement procedures \\ 6a. Identifying and avoiding medico-legal risks in complete denture prosthetics \\ 6b. Identifying and avoiding medico-legal risks in removable dentures \\ 7. Dento-legal aspects of orthodontic practice \\ 8. Temporomandibular disorders \\ 9. Dental implants \\ 10. Periodontology \\ 11. Oral surgery}

Specialist Orthodontist, 128 Woodford Avenue, Gants Hill, Essex, IG2 6XA/Associate Specialist, Department of Orthodontics, Whipps Cross University Hospital, London

Correspondence to: Dr Eliakim Mizrahi Email:kimmizrahi@aol.com

\section{Refereed Paper}

Accepted 20 October 2009

DOI: $10.1038 /$ sj.bdj.2010.926

${ }^{\circledR}$ British Dental Journal 2010; 209: 381-390 decade, started to take on significant dimensions, particularly in the United States. A comprehensive discussion on risk management in orthodontics is presented in a book edited by Graber, Eliades and Athanasiou. ${ }^{1}$ While the incidence of malpractice litigation in the United Kingdom may be at a lower level, it is nevertheless an important issue that concerns the General Dental Council, all professional bodies, associations, insurance companies and particularly all dental practitioners. Over the last five years, of all the cases addressed by the Professional Conduct Committee of the General Dental Council, $2.9 \%$ were related to orthodontics. While this figure may be considered low, it does not reflect the cases that involve litigation but do not progress to a disciplinary hearing of the General Dental Council; this information is not available.

The overriding principle in risk management is 'anticipation and avoidance'. Clinicians need to make every effort to avoid getting into situations that may lead to litigation. A number of issues involved in this process are common to the entire discipline of dentistry, however there are features of risk management that are more pertinent to orthodontics; these will form the basis of this article.

Orthodontics is unique in that treatment generally extends over 18 to 24 months. This presents the orthodontist with an opportunity to establish a pleasant, friendly, empathetic and special relationship with their patients. Such a relationship is the best antidote to any possible future litigation. As a general rule, patients who have a good personal relationship with their clinician are less likely to litigate as opposed to those patients who are upset and feel resentment against their dentist. When physicians who had never been sued were polled about what they thought the reason was, they all gave exactly the same answer independently: a strong doctorpatient relationship. ${ }^{2}$

Evaluating risk assessment in orthodontics can be simplified by classifying the patient-orthodontist relationship into three periods: pre-treatment, treatment and post-treatment (Fig. 1) However, irrespective of the treatment period there are certain concepts that apply throughout the orthodontic experience:

- Establish a good professional relationship with your patient; pitch your relationship and level of conversation to match their age

- Be empathetic, try to understand how it feels to be at the receiving end of your hands and instruments and apologise often during an uncomfortable procedure

- Smile, greet your patients by name and try to be pleasant throughout their orthodontic experience 


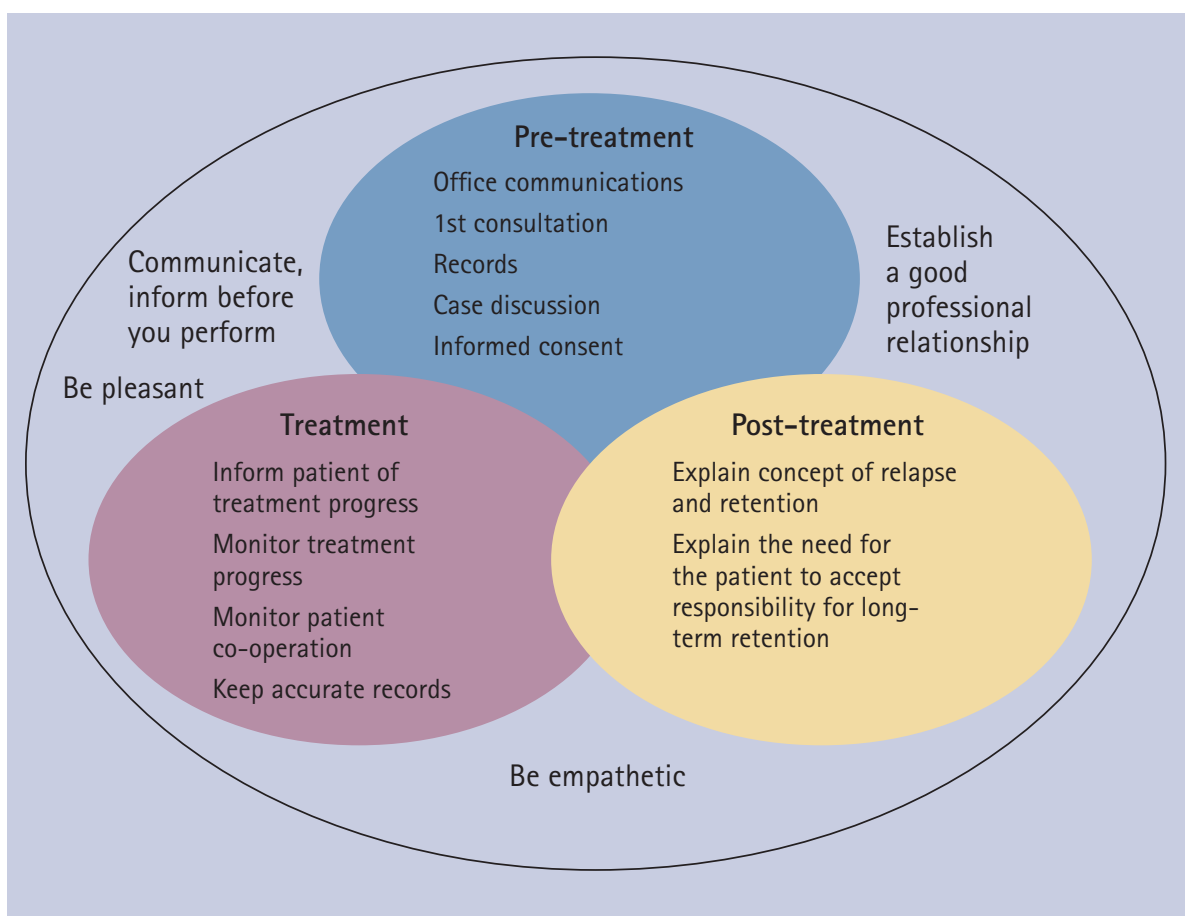

Fig. 1 Diagrammatic representation of pre-treatment, active treatment and post-treatment time periods, showing potential risk management issues

- Communicate and 'inform before you perform'. Patients and parents want to be informed and more importantly they have a right to know in advance what procedures will be performed.

\section{PRE-TREATMENT PERIOD}

In the context of the pre-treatment period, patient-orthodontist contact involves the first consultation, record taking and case discussion. The first contact between the patient and the practice may be the initial phone call, a receipt of the practice brochure or a first visit to the surgery/office.

From any of these contacts the patient will form an impression of the practice either via verbal communications or visual perceptions. It is essential that whatever information is imparted at this stage, it should be true and realistic. The patient should not be given information that cannot stand up to scrutiny by a third party and the patient should not be given information that leads him or her to have expectations for treatment that are unachievable. ${ }^{3}$

At the first consultation the patient/ parent should be informed of the initial findings, given an idea of what procedures will be involved, an explanation regarding the need for investigations/records as well as their cost and if possible an idea of different appliance systems. Patients will generally ask for an estimate of the treatment duration and a possible range for the overall cost for treatment. The patient should be informed that before having examined all the relevant records, it is not possible or wise to be specific; an estimate of time and cost may be given but more precise information will be provided at the case discussion and followed up with a confirming letter.

Specialist orthodontists, having undergone postgraduate training, are generally equipped to treat most malocclusions, however a number of general practitioners with a special interest in orthodontics also treat a range of malocclusions. It is important and prudent for each clinician to know his or her limitations. Unfortunately, no matter how well trained or experienced an orthodontist may be, every clinician has some failures and nobody is infallible. It may be difficult, however, to defend a legal claim for negligence against a clinician who has failed to treat a case adequately when it is established that his or her training does not match up with that required for the treatment of the malocclusion in question.

It is the responsibility of a general dental practitioner to refer a patient at the most appropriate time if they do not provide comprehensive orthodontic care themselves. The timings of these referrals vary and in themselves may be the subject of some dento-legal risk.

Crucial stages are between seven and nine years old when general dental practitioners need to be looking out for problems such as:

- Anterior or posterior cross bite

- Space maintenance after premature loss of deciduous molars

- Thumb sucking habit intervention

- Compromised first permanent molars

- Developing impaction of unerupted permanent teeth

- Developing Class II and Class III malocclusions.

In 11-12-year-old patients general dental practitioners need to consider the importance of extracting retained deciduous teeth to allow their permanent successors to erupt. When the permanent tooth has erupted on one side of the arch and not the other it is important to establish the reason. This is also a crucial time to assess the position of the maxillary canines. In the case of palatally placed permanent canines, the extraction of the deciduous canine between ten and 13 years old is in certain cases an effective treatment approach and studies have shown that this can be $91 \%$ successful if the unerupted canine overlaps the lateral incisor by less than half the breadth of the root when visualised on a panoramic radiograph. ${ }^{4}$ The issue of early treatment for Class II and Class III malocclusions is contentious, and falls outside the scope of this article; nevertheless, in the best interests of the patient, the general dentist should refer the patient and leave the treatment decisions to the orthodontist.

At the first consultation it is important to ascertain the motivation and expectations of the patient or parent even before a full investigation and if their expectations are unrealistic or cannot match the ability of the clinician to deliver and satisfy the patient, this needs to be pointed out and treatment refused and possibly referred elsewhere. If at the end of the first consultation, the clinician is happy to proceed with the next step, the necessity for records as well as their cost should be carefully explained and a mutual decision taken to proceed with the investigations. As good practice dictates, this should all be noted on the record card. 


\section{Orthodontic investigations}

At the appointment scheduled for taking all the necessary records, there is as yet no written consent. However, the fact that the patient has arrived and is aware of the procedures and costs that will be incurred, eg impressions, radiographs and photographs, can be taken as implied consent.

\section{Clinical examination}

A clinical examination covering an evaluation of extra-oral and intra-oral hard and soft tissues is essential. It is not within the scope of this article to detail the requirements of a comprehensive clinical examination, diagnosis and treatment planning; these are well documented in many textbooks and publications. ${ }^{5-7}$ Suffice to mention that all procedures should be adequately documented either as a hard copy or in digital format. A comprehensive medical and dental history is mandatory.

With regard to risk management, there are certain elements that require special mention:

- Temporomandibular disorders (TMD)

is a collective term for a number

of clinical problems involving

the masticatory musculature, the temporomandibular joint (TMJ) or both. Despite the enormous volume of literature, lectures and courses on the subject, a clear and complete understanding of all facets of TMD is currently lacking. The frequency of severe disorders is $1 \%$ to $2 \%$ in children, 5\% in adolescents and 5\% to $12 \%$ in adults. ${ }^{8}$ Based on this evidence, orthodontic practices receive many patients who present with TMD signs and symptoms regardless of their main complaint. Evaluation of the health of the TMJ must be carried out as part of the clinical examination and both positive and negative findings recorded. If positive signs such as clicking are found, depending on the severity, an assessment as to the treatment options needs to be established. In cases where the patients have been unaware of any clicking, crepitus or pain, they should be told of the findings and informed that there is no current contra-indication to orthodontic treatment, but they should be aware that the condition may either remain the same, improve or deteriorate at any stage during and/ or following orthodontic treatment. In cases where the symptoms are severe and the patient is experiencing pain, this condition must be addressed before starting orthodontic treatment. The clinician needs to decide as to his or her ability to treat the condition or refer to a more suitably qualified or experienced colleague. TMD problems are difficult to treat, their outcome is often below expectation and they have been used as a reason for instituting legal action. It is essential that comprehensive notes are recorded and communicated to the patient orally and in writing

- Periodontal disease. Given the number of claims related to periodontal treatment, orthodontists should be knowledgeable about the periodontal implications of orthodontic treatment. Gingivitis produces reversible lesions which, with improved oral hygiene, can be resolved. However, adult periodontitis is an irreversible lesion. It causes loss of attachment and supporting alveolar bone. ${ }^{9}$ Both conditions may present in either adolescent or adult patients, however periodontitis is more likely to present in adult patients. For all patients, the gingival and periodontal condition needs to be evaluated at the clinical examination with the aid of a periodontal probe and radiographs. Record all findings. If the oral hygiene is poor, the patient, parents and referring dentist need to be made aware of the problem and the probable consequences. Many adult patients presenting for orthodontic treatment have mutilated malocclusions associated with periodontal disease. While they are aware of their unsightly teeth, tragically, in spite of regular dental attendance, they are often unaware of their periodontal problem. With the consent of the referring dentist, these patients must be referred to a periodontist for consultation and treatment (Figs 2a and b and $3 \mathrm{a}$ and $\mathrm{b}$ ). Orthodontic treatment should only be commenced when the periodontist confirms in writing that the disease is stable and under control. At the start of orthodontic treatment, the patient

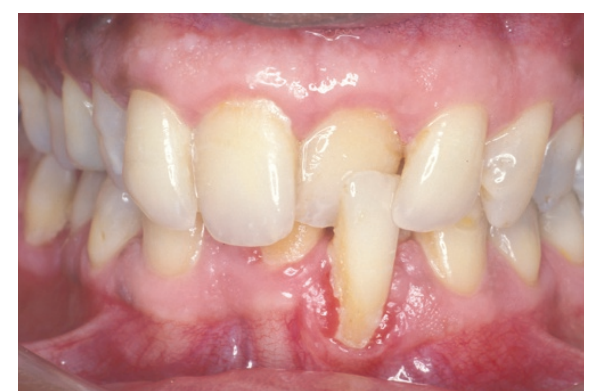

Fig. 2a Class I. Crowded, anterior crossbite, poor oral hygiene, gingival recession and loss of supporting alveolar bone

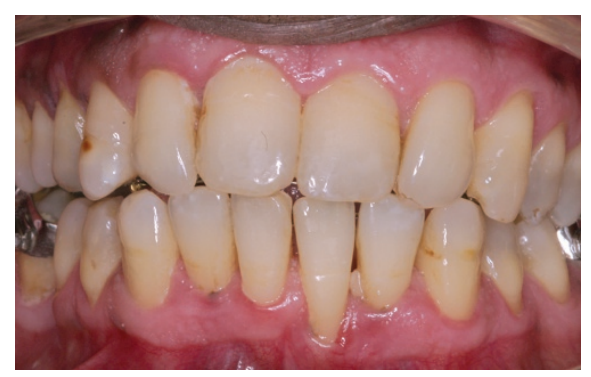

Fig. $2 \mathrm{~b}$ Treatment progress showing improvement in alignment and gingival inflammation (lingual appliance)

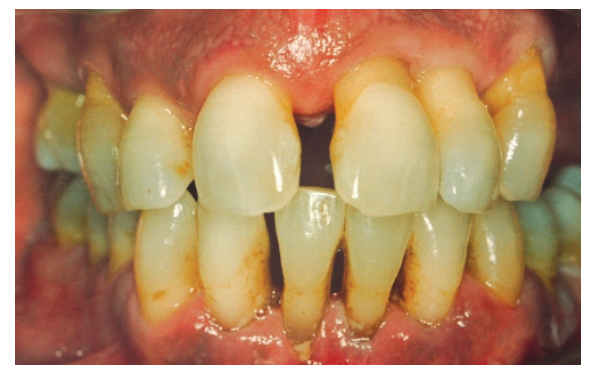

Fig. 3a Pre-treatment Class I. Chronic periodontitis with loss of supporting alveolar bone

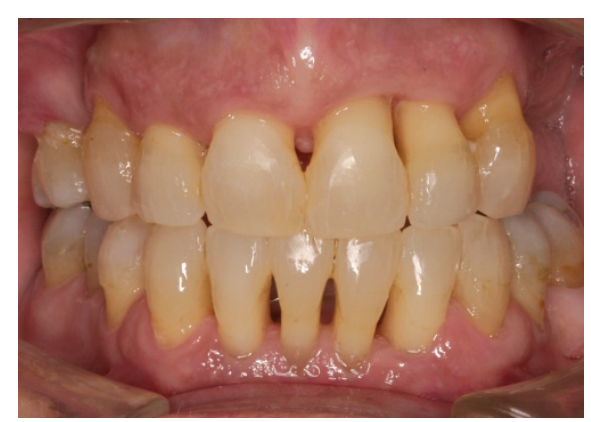

Fig. 3b Post-treatment following periodontal therapy and orthodontic treatment

must be informed orally and in writing that they need to continue to see the periodontist throughout treatment and that in spite of the disease being stable, there is still a possibility of further bone loss during treatment

- Trauma to jaws or teeth. A history or clinical evidence of trauma is an 


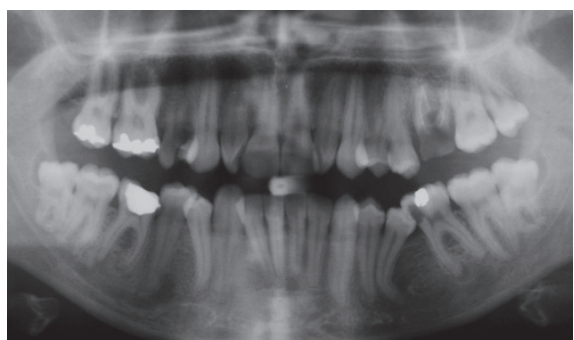

Fig. 4a Pre-treatment radiograph showing gross caries

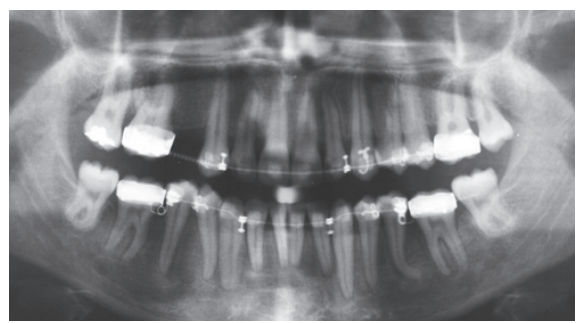

Fig. 4b Progress radiograph following extraction of carious teeth and subsequent tooth alignment

essential component of the clinical examination. Clinical evidence of chipped or cracked teeth must be recorded and it should be explained to patients or parents that once teeth have had a blow sufficient to cause a chip or a crack, the nerve may be dead or may die gradually over a period of time. Clinically this may present as a grey discolouration either immediately or gradually over the treatment period. It is important for them to understand and appreciate that such changes are not as a direct result of orthodontic treatment but are the sequel to a traumatic episode. Should the tooth become non-vital during treatment they must be aware that it will need to be root treated. Previously root treated teeth need to be evaluated for soundness of the root filling, apical pathology and root structure. Particularly in the case of younger patients, anterior teeth may have a history of partial fracture, partial avulsion or full avulsion and re-implantation. The prognosis for these teeth may be uncertain and this information should be imparted to the patient and parents. It is expected that adequate tests for pulp vitality and root ankylosis are carried out. It is important to establish the history of previous major trauma, surgery or pathology to the cranio-facial elements. Trauma to the mandible may be related to TMJ dysfunction, may have resulted in condylar fractures and mandibular deviations or asymmetry. Neoplasms of the orofacial region that may have received radiation therapy will have had an effect on bone vascularity and possible inhibited tooth movement

- Caries and apical pathology. The caries status of the dentition should be assessed and if necessary any restorative work completed before starting orthodontic treatment. If apical pathology is apparent on radiographs before starting treatment, then the patient needs to be referred back to his or her general dentist for any necessary endodontic treatment before starting orthodontic treatment (Figs $4 \mathrm{a}$ and $\mathrm{b}$ ). It is not uncommon for adult patients to present with a root treated tooth associated with an apical translucency that has been present with no symptoms for many years. This issue needs to be discussed with the patient and the general dentist. It may be acceptable to proceed with orthodontic treatment provided the patient has been informed that this tooth may react unfavourably to tooth movement at any stage during orthodontic treatment and would then need endodontic treatment

- Root resorption. An in-depth discussion of this subject falls outside the scope of this article, however it is an area of major concern for orthodontists because of the clinical and legal implications associated with its occurrence. ${ }^{10}$ There are no truly reliable predictive factors and the exact nature of the initiation and control of apical root resorption remains essentially unknown. ${ }^{11}$ Assessment of the risk of root resorption starts with the patient's medical and dental history to evaluate the possible predisposing influence of hereditary, systemic and local factors. If any relevant predisposing factors become evident during the clinical examination, then further radiographic evaluation using standardised periapical radiographs taken with a film holder needs to be carried out. A study by Levander and

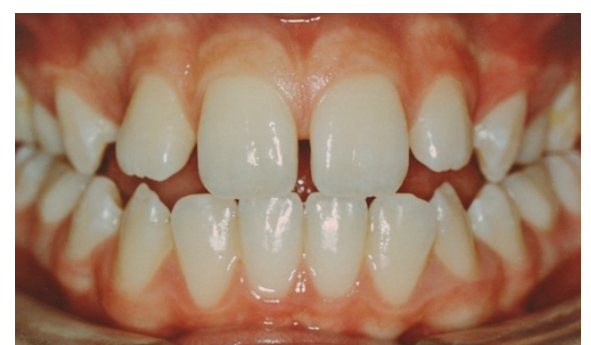

Fig. 5a Class I malocclusion with early loss of maxillary deciduous canines and displaced lateral incisors

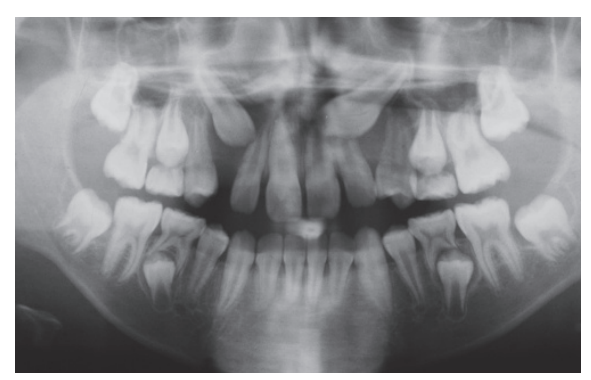

Fig. 5b Panoramic radiograph showing impacted canines and root resorption of lateral incisors

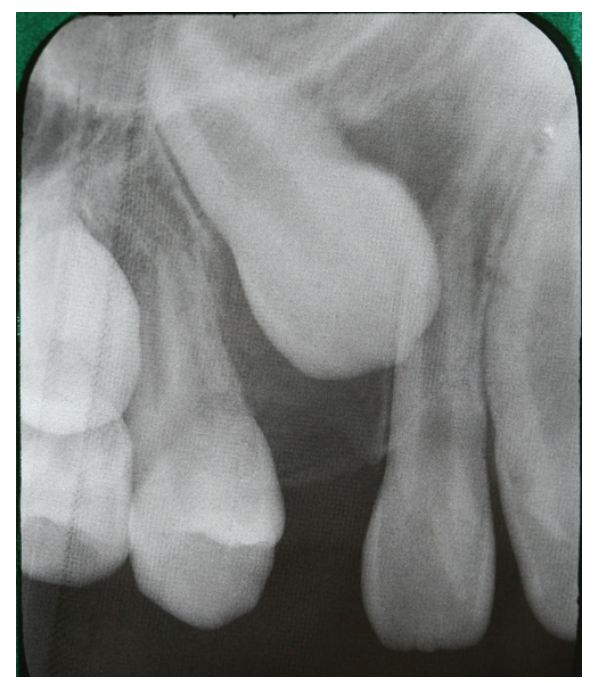

Fig. 5c Periapical radiograph showing impacted 13 (UR3) in contact with the root of the 12 (UR2)

Malmgren showed that the degree of root resorption in teeth with blunt or pipette shaped roots was significantly higher than in teeth with a normal root form. ${ }^{12}$ The clinical and radiographic evaluation of unerupted and impacted teeth, particularly canine teeth, is essential in view of their association with root resorption of adjacent teeth. Using conventional radiography root resorption of lateral incisors adjacent to impacted canines occurred in $12 \%$ of patients, however with computerised tomography (CT), root 


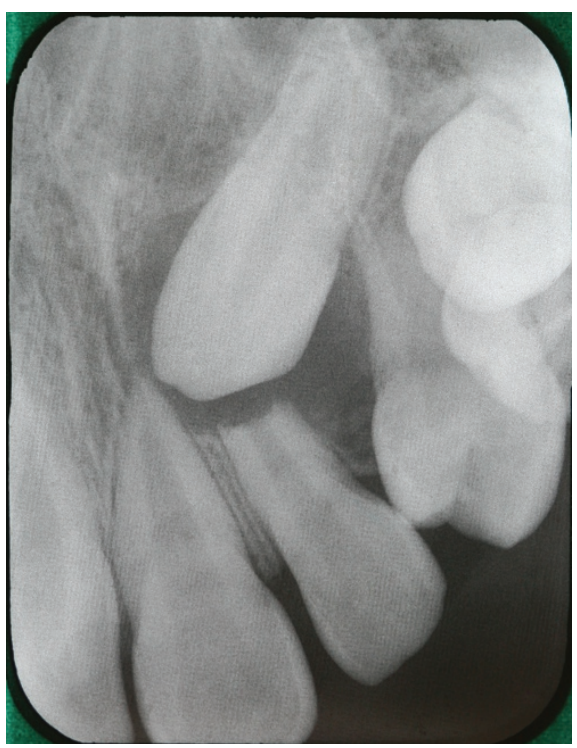

Fig. 5d Periapical radiograph showing impacted 23 (UL3) and root resorption of 22 (UL2)

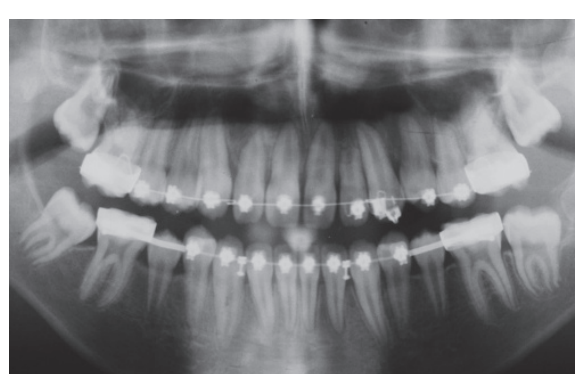

Fig. 5e Panoramic radiograph showing final alignment and arrested root resorption of lateral incisors

resorption was shown to occur in 38\% of lateral incisors and 9\% of central incisors (Figs 5a-e). ${ }^{13,14}$ Any evidence of the potential for root resorption developing during treatment must be communicated to the patient or parent, recorded and taken into account in treatment planning with, if possible, shorter treatment time, minimal forces, and limited goals. A note should be made at this early stage to monitor the root condition throughout treatment with progress radiographs to be taken after six to nine months

- Oral hygiene. Poor oral hygiene at the clinical examination is often an indicator of poor oral hygiene during treatment; this applies to both young and adult patients. These patients need to be monitored carefully for plaque, gingival inflammation and the development of enamel decalcification. Patients must be advised verbally and in writing of their responsibilities, orthodontist's expectations and the consequence of non-compliance. Caries and decalcification are issues that can lead to claims and litigation. It may be useful to record plaque scores after using disclosing tablets with accompanying photos recording this status at the start of treatment. A session with an oral health educator or orthodontic auxiliary may be useful for at-risk patients.

\section{Study models}

Apart from their contribution to diagnosis and treatment planning, study models form an essential record of the pre-treatment status of the dentition and are an invaluable aid in the defence of any litigation that may arise in the future. Neither the clinician nor the patient can reliably recall the original status of the dentition two or more years later.

While historically study models have always been cast in hard plaster, currently there are sophisticated techniques that make use of CADCAM technology to produce digital study models; a major advantage of this technology is the ability to store this form of record in digital format.

\section{Photographs}

As with study models, good photographs are an aid to diagnosis and treatment planning as well as contributing to the record component required in any litigation situation that may arise. Not only do photographs provide a record of the malocclusion, they also provide a good record of colour blemishes, mottling, decalcification and staining, all issues that could be a source of future litigation. It should be part of routine orthodontic practice that pretreatment photographs and study models are taken and retained.

\section{Radiographs}

There is a general awareness by the profession and informed patients of the need to reduce, to a minimum, exposure to ionising radiation. This limitation impacts on the use of radiographs in orthodontics. The guiding principle for taking orthodontic radiographs as put forward by the British Orthodontic Society is "No patient should be expected to receive additional radiation dose and risk as part of a course of dental treatment unless there is a benefit in terms of improved management of the patient. ${ }^{15}$ There is no question regarding the need for adequate good quality pretreatment radiographs taken to assist in diagnosis and treatment planning. The debate centres on the practice of taking progress radiographs during treatment and taking final radiographs at the end of treatment. Before taking a radiograph the clinician needs to ask 'will this radiograph be of direct benefit to the patient?' Bearing this issue in mind, it is essential to examine the reasons for taking progress and final radiographs.

Progress radiographs are taken to assess a number of issues:

1. Root angulation, particularly on either side of extraction sites as well as root torque of anterior teeth, although it may be argued that the position of these teeth can be adequately assessed by careful clinical examination

2. Probably of greater importance is the need to assess developing root resorption and possible loss of supporting alveolar bone. In a young healthy dentition, with no initial evidence of predisposing root resorption or alveolar bone pathology, it would be rare for these conditions to manifest during treatment thus possibly contraindicating the need for a progress radiograph. However, if pre-treatment radiographs show evidence of pre-existing root resorption or if, as described earlier, the root morphology indicates a risk of developing root resorption, then these patients should have progress radiographs taken at six to nine months. ${ }^{12,16}$ With adult patients, the loss of alveolar bone and possible root resorption are risk factors that need special consideration and warrant the taking of progress radiographs

3. Irrespective of age, for patients presenting with impacted teeth, whether these are exposed, extracted or left in situ, the risk of root resorption or cystic development is increased and warrants periodic radiographic monitoring

4. The possible development of caries during treatment is a consideration. The oral health of the patient should have been evaluated at the start of treatment and any carious lesions 
attended to before the commencement of treatment. However, if carious lesions develop during treatment or if the patient complains of pain for which there is no clinically obvious reason, then radiographs of the relevant region would be indicated

5. Radiographic images of the temporomandibular joint require special imaging techniques and should be evaluated by individuals trained in this field. While certain orthodontists may have trained and may have the experience to correctly evaluate these special images, many do not and would feel more comfortable referring patients with TMD to specialists in this field. Should a dento-legal issue arise it would be incumbent on the clinician to justify the radiographic images taken.

Final radiographs. In the past, taking final panoramic and cephalometric radiographs was considered essential and good practice. Currently, taking final radiographs is controversial. Many retrospective and prospective studies were and still are being carried out on the data accumulated from pre- and post-treatment radiographs and much of orthodontic theory, technique and research is based on information gleaned from these studies. However, what is questionable is whether the patient actually benefited directly from these radiographs? If the radiograph is taken as the end of treatment approaches, and there is still an opportunity to correct or improve the final root position, then it may be possible to justify the radiographic exposure. A prefinal radiograph also gives the clinician the opportunity to evaluate possible root resorption, alveolar bone levels and the status of any unerupted teeth such as the third molars. Although it may be too late to institute possible preventive measures, it does give the clinician the opportunity to inform the patient that such abnormal changes have taken place and inform them of possible future developments.

Having a radiographic record of the near final position of the teeth, jaw relationships, bone condition and soft tissue drape does give the clinician some degree of protection with regard to possible future legal claims that may be instituted by the patient.

\section{Case discussion}

This is probably the most important appointment; it is at this appointment that the patient/parent will have the case explained to them in a language that they can understand, have a treatment plan presented, have a discussion of alternative treatment options, and finally presented with a fee structure if appropriate. The concept of retention also needs to be explained as well as the fee structure for this phase of treatment.

It is essential to understand and appreciate patient expectations and to marry them with possible limitations of orthodontic treatment. In many cases final objectives may need to be realistic as opposed to idealistic, particularly in adult cases with skeletal discrepancies, mutilated malocclusions and periodontal problems. Explain in simple terms what can and what cannot be achieved in relation to the patient's expectations.

The issue of potential risks of orthodontic treatment is contentious. The question that arises is, is it necessary to discuss all the potential risks no matter how small the risk is? Or should you only mention and discuss the possible risks that may be evident/apparent from the initial records? There is no doubt that if there is clinical or radiological evidence of pre-disposing factors such as TMJ symptoms or evidence of pre-existing root resorption, periodontal disease or high caries susceptibility, then it is essential that this issue be explained and discussed at the case discussion. If on the other hand there is no clinical or radiological evidence of any pre-disposing factors then I believe it is sufficient to inform the patient/parent that at present there are no apparent risk factors that need elaboration but that a list of potential risks associated with orthodontic treatment will be mailed to them with a letter confirming the information presented at the case discussion. Should they then have any queries or concerns, they should not hesitate to call and discuss the matter. If pre-disposing risk factors have been identified, it is essential to explain to the patient the advantages or disadvantages in proceeding with active orthodontic treatment.

Multidisciplinary treatments involving possible referral to another specialist such as a periodontist or maxillofacial surgeon should be discussed and arrangements for such a referral documented and set in motion. There should be appropriate liaison between clinicians and clarity about who is providing what treatment. This is particularly relevant where spaces are being retained for the placement of bridges or implants. The patient should be given sufficient information about risks and costs to be able to consent to both the orthodontic treatment and the associated treatment, before either treatment is started.

Examples of issues which may predispose to further complications and which may require referral are unerupted and impacted teeth, existing gingival recession, gingival inflammation, pocketing and loss of alveolar bone, high caries rate, and TMJ symptoms. The British Orthodontic Society has produced an Advice Sheet (number 23) on the risks of orthodontic treatment and guidance on informing patients.

If the case presents with crowns or veneers, the patient should be warned that during orthodontic treatment there may be changes to the gingival margins or damage to the porcelain surface at debonding, either of which may require the crown or veneer to be redone by their general dentist.

Throughout the case discussion session encourage the patient to ask questions. At the end of the case discussion, inform the patient/parent that they will be receiving a letter covering in brief terms what has been discussed together with a list of potential risks associated with orthodontic treatment as well as an informed consent form which they should sign and return after having read and understood all the documentation.

\section{Letter to the patient/parent}

The letter confirms the discussion: ${ }^{17}$

- Describe the skeletal pattern in layman's terms, '...has an acceptable or protrusive or retrusive lower jaw in relation to the upper jaw...'

- The state of the dentition; mention conditions such as crowding, spacing, protrusion and any other relevant features

- The proposed treatment plan, mentioning the type of orthodontic appliances, if necessary, the need or possible future need for extractions

- Where relevant, the need to consult with another specialist 
- The approximate duration of active treatment

- The importance of retention

- The proposed costs including the method of payment

- Other items such as the need to continue visiting their general dentist, the need for co-operation with regard to appliance care and maintenance of good oral hygiene

- Currently it is considered good practice to include, either as a separate enclosure or as part of the letter, a listing of the possible hazards and risks associated with orthodontic treatment

- As confirmation of having read and understood your letter, a request for the patient or parent to sign and return an enclosed consent form.

\section{Letter to the dentist}

Following the case discussion a letter needs to be sent to the patient's dentist providing relevant information about the case. If teeth need to be extracted, it is essential that this is indicated very clearly. There are different international systems for identifying teeth; the orthodontist and general dentist need to be on the same system. As a safeguard consider using the FDI system followed by a further identification in brackets, eg tooth 23 (UL3). Extraction of the wrong tooth can become a malpractice issue. If the information conveyed to the clinician carrying out the extraction is clear and unambiguous, any mistakes will be the legal responsibility of the treating dentist, not the orthodontist.

Requesting an extraction via telephone is a risky procedure; any such request must be followed up with a confirming letter. It is the responsibility of the dentist taking the tooth out to ensure that the correct tooth is extracted. If there is any doubt, the orthodontist should be contacted. Every year, many incorrect extractions are carried out by general dental practitioners following referrals back from the orthodontist. This may occur simply due to carelessness on the part of the dentist removing the tooth but on occasion it may be the fault of the orthodontist or the typist. In some cases the incorrect extraction could result in the treatment simply taking longer to complete, for example if a first premolar is taken out instead of a second premolar. Occasionally the wrong deciduous tooth is taken out which has no permanent successor. In that case, if residual space remains after orthodontics, the patient may require implants or a bridge for which they would be entitled to seek compensation from the dentist who extracted the wrong tooth. A breach of duty has occurred and harm has followed. Irrespective of where the original fault lies, the patient/parent needs to be informed of the occurrence.

\section{Consent form}

It is essential for a parent or adult patient to sign a consent form before starting any orthodontic treatment. Most national orthodontic societies have a recommended consent form. In view of the variation in scope and complexity of such a form, it makes sense to adopt the form recommended by your national orthodontic society. A clinician may be negligent in not providing relevant information about risks before the patient gives consent to a procedure. The essential prerequisite is that the patient or parent should not only sign but should also understand what they are signing. The signed original or copy must be kept with the patient records. ${ }^{17}$ It is interesting to note that while parents and patients recall signing a form, they sometimes fail to recall certain crucial information such as the risk of treatment and the need to wear retainers for a protracted period of time. ${ }^{18}$

\section{ACTIVE TREATMENT PERIOD}

During the active treatment phase the overriding considerations are monitoring treatment progress, keeping the final goal in sight and keeping control of the treatment duration. Whatever complications may or may not arise during treatment, the essence of good practice, and the best protection for the clinician should any legal issues arise, is the keeping of good comprehensive legible records either written on a record card or recorded in digital format. At every appointment an accurate and legible note should be made of all procedures carried out, as well as noting any untoward developments. Untoward conditions related to treatment mechanics such as dental pain, excess tooth mobility, gingival inflammation and temporomandibular joint dysfunction, as well as conditions related to patient co-operation such as poor oral hygiene, the development of decalcified lesions, failure to wear elastics, excess appliance breakages and failure to keep to appointment schedules, should all be detailed in the record notes. Where there are issues related to patient compliance that have not been resolved by direct discussion with the patient or parent, then these should be documented in a letter to the patient or parent.

Breakage and loss of some components of the appliance may occur at any stage during active treatment. These episodes need to be carefully recorded. It is important to establish from the patient whether the bracket or piece of wire was swallowed, removed from the mouth or inhaled. Unfortunately, in many cases the patient is not aware that anything is missing, or does not know what happened to the missing piece of hardware. An item such as a missing bracket is unlikely to have any untoward effect and will in due time probably pass through the gut. However, a piece of wire one or two centimetres in length may have more serious consequences. In such cases, the patient should be informed of the occurrence, warned to be alert to any possible symptoms developing and should be referred to their medical practitioner. Does it warrant taking abdominal radiographs? This decision should be taken in conjunction with the patient's medical practitioner after balancing the hazards of exposure to ionising radiation with the chance of identifying on a radiograph a thin piece of stainless steel wire 1-2 cm in length anywhere in the abdominal cavity. Many small objects such as brackets and auxiliary springs are used in fixed orthodontics; these may be swallowed or inhaled and should be managed appropriately. ${ }^{19}$

The risks involved in wearing headgear and the need to warn patients of theses risks have been widely reported in the literature. ${ }^{20}$

Accidental injuries in the surgery are not common but can occur at any stage of treatment. It is accepted that protective glasses are provided for each patient for every procedure. However, there is still the possibility of spilling etchant on the soft tissues, lacerating the lips or tongue or a clipped piece of wire may jump into the eye in spite of the protective glasses. If an accident does occur, the patient must receive whatever medical care is needed whether from the dentist, a nearby doctor or at 
a medical emergency unit. If the patient requires transport, it should be arranged by the clinician. The cost for any emergency treatment should be borne by the clinician responsible; financial concerns can create animosity that encourages claims and litigation. Empathy is an important factor in these incidents and a call by the orthodontist to enquire about the well-being of the patient is good practice. At the time of the incident, emotions of all concerned will be high, the orthodontist may not be clear about all the facts that precipitated the incident and should not admit liability under any circumstance. The insurer must investigate and determine liability once the claim is reported..$^{21}$

\section{Debonding}

During debonding of porcelain brackets it is possible to chip or fracture the labial enamel. If this does occur, the patient must be informed and if necessary arrangements made for the required repair procedures.

\section{Terminating treatment early}

Non-compliance and poor co-operation, periodontal disease, root resorption, caries, financial delinquency and a breakdown in patient-clinician relationship are all factors that may contribute to the decision to terminate treatment early. This decision must be taken after much deliberation and discussion with the patient/parent and supported by adequate documentation. When patients feel rejected, they often retaliate and institute legal proceedings by alleging either treatment-related problems or patient abandonment.

Before terminating treatment early, consider what effect this will have on the patient with regard to the state of the occlusion, the oral health and the psychological impact. The legal implications need to be assessed in conjunction with the original documentation provided to the patient.

\section{POST-TREATMENT PHASE}

As the end of active treatment approaches, it is prudent to explain to the patient or parent that the teeth are almost at their final position and they need to look in the mirror in their own time and make sure that they are happy with the alignment of the teeth. If there is a tooth that they feel needs further correction, this is the time to make adjustments, not after the appliances have been removed. Extending the treatment by one or two appointments can make the difference between a happy and unhappy patient. Giving a child or adult patient the opportunity to have some say or input in the final stages of the treatment gives them a sense of importance and satisfaction and contributes to achieving a good result and a happy patient. Remember that a happy patient is unlikely to ever institute legal action. Generally the patient is unaware of the occlusion and the alignment of the posterior teeth; it is the responsibility of the clinician to achieve the best possible occlusal and functional result.

While a careful clinical examination of all the teeth and supporting soft tissues is essential throughout treatment, it is particularly important to evaluate the status of the dentition as the end of active treatment is approached. Not every case will always work out to be perfect; there are many issues which preclude the achievement of a perfect result. Limitations imposed by the skeletal pattern, soft tissue drape, the status of the dentition and patient compliance may to varying extents contribute to a less than perfect result. Some of these limitations would have been noted at the original case evaluation and explained at the case discussion, however if one or more issues only became apparent during treatment, then the issue in question should have been explained to the patient or the parent and they should be aware that the end result may be compromised. If at the end of treatment the final result is not as good as it should have been, it is important to point this out to the patient or parent and generally, if the deficiency is explained, they will trust and accept your explanation. However, if nothing is said at this stage, and the deficiency is later pointed out to the patient by their general dentist or some another clinician, then the patient may well feel aggrieved and unhappy and this in turn may lead to a possible litigation scenario.

I believe it is prudent to take a progress panoramic radiograph at this stage. As mentioned previously the progress radiograph will provide information on a number of dental and skeletal issues that both the patient and clinician should be aware of before the completion of active treatment. Of particular note at this stage

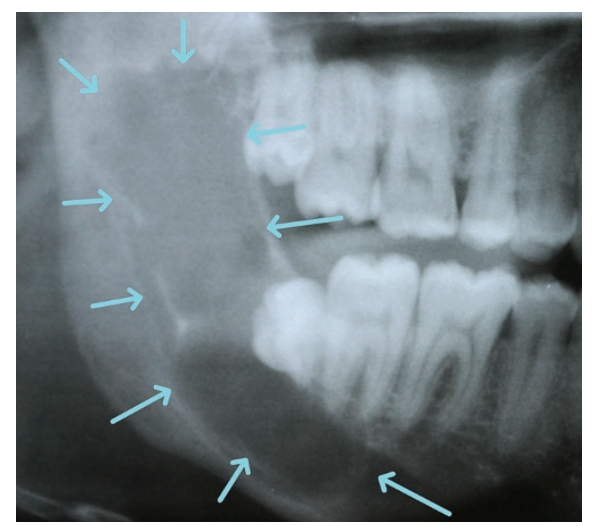

Fig. 6 Large bone cyst associated with an unerupted 48 (LR8)

is the presence of unerupted and impacted third molars. A discussion on the advisability of extracting impacted third molars falls outside the scope of this article. However, it is important to point out to the patient or parent that these teeth are impacted and they may pose problems at a later date (Fig. 6). The important issue here is that the patient is aware of the potential problem and that it has been recorded on their card.

At this stage it is essential to explain to the patient and parent that following removal of appliances, it will be necessary to fit retainers. Remind them that the concept of retention was explained to them at the start of treatment and at this stage you are refreshing their memory. Patients have short memories and it is necessary now to once again explain the reasons for retention, the type of retainers that will be fitted and more importantly, explain your philosophy on the duration of retention. The duration of retention is controversial; there are no evidence-based data that dictate exactly how long teeth should be retained, however what all orthodontists do know is that teeth move throughout life. Based on this premise and knowing that certain malocclusions are more susceptible to relapse than others, each clinician inevitably has his/her own opinion as to the duration of retention. For many adult patients, because of skeletal restraints and profile considerations, teeth are often placed in an unstable position. In these cases retention is for an indefinite period of time. The patient needs to understand and be aware of this concept (Figs 7a-e). Apart from the time span for retention, a further controversial consideration is the responsibility factor. At 


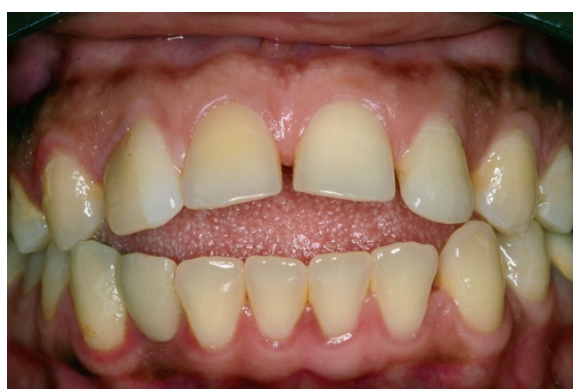

Fig. 7a Anterior open bite; excess space filled with composite on distal of 12 (UR2) and pontic mesial to 43 (LR3)

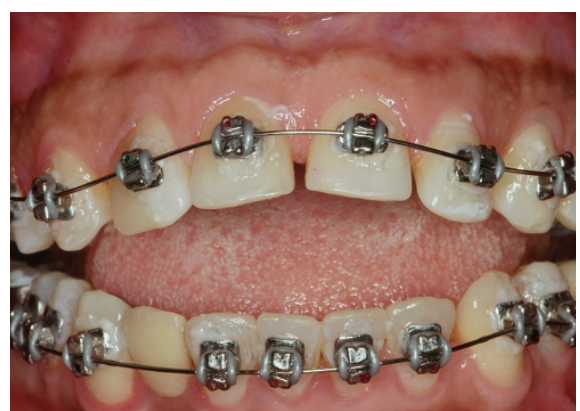

Fig. $7 \mathrm{~b}$ Fixed appliances to reduce anterior open bite and close all spaces

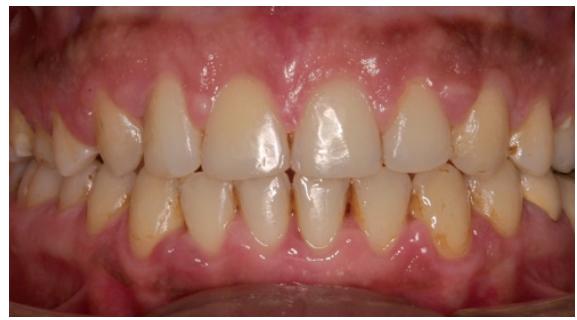

Fig. 7c Anterior open bite reduced and all spaces closed

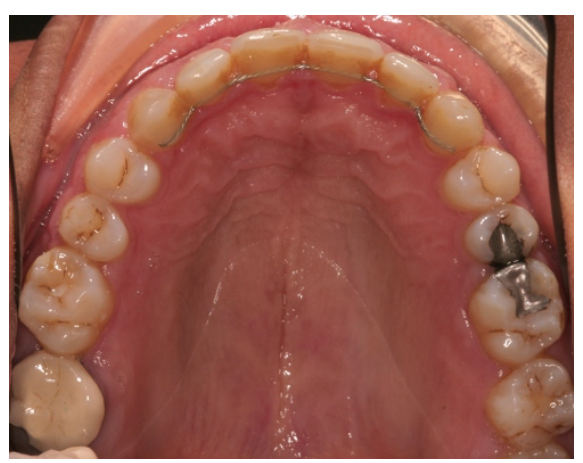

Fig. 7d Fixed maxillary palatal retainer

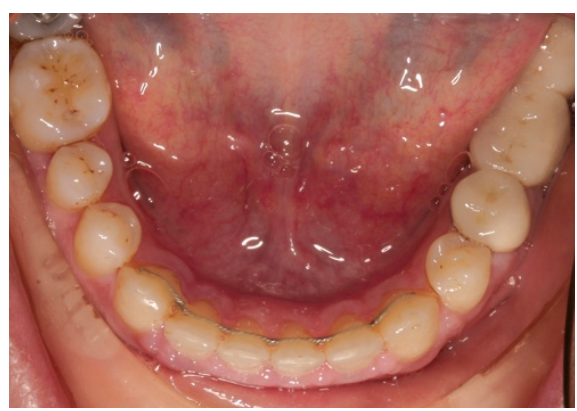

Fig. 7e Fixed mandibular lingual retainer some stage responsibility for the care and maintenance of the retainers must move from the clinician to the patient; it is essential to explain and provide written instructions on how the appliances must be cared for, how they must be worn and for how long they must be worn. Explain that whether the appliance is fixed or removable, they are all potential food traps and if neglected, they can cause damage to both the teeth and supporting gums. Once your period of observation is over, they must continue to see their general dentist and if necessary they should feel free to return to you for a consultation. The financial arrangements for retention and post-retention observation need to be explained and confirmed before appliances are removed. If the fee for retention was not included in the original quote for active orthodontic treatment, this should have been explained at the case discussion and noted in the confirming letter to the patient or parent. If a separate fee is charged for retention, then this fee must be quoted before appliances are removed. Once again, to keep a patient happy, do not spring financial surprises on them. All the above procedures need to be entered on the patient's record card.

\section{Mandibular growth}

Mandibular growth after treatment can pose a problem, especially in males. Continuing growth in either the horizontal plane (Class III) or the vertical plane (anterior open bite) may disturb the final orthodontic result and the parents may then allege poor orthodontic diagnosis and treatment. To counter this allegation, the possible effects of post-treatment growth should have been pointed out at the original case discussion and the availability of final study models can assist to prove the treatment outcome before the growth took place. Claims develop when the parents are not adequately apprised of all aspects of their child's dental care, especially if it relates to the need for surgery.

\section{Refunding fees}

This is an important claim-minimising issue that has advantages and disadvantages. Each situation must be considered on its own merits taking into account the patient and/or parents, the treatment issues, the problems alleged and the amount of money involved. If a refund is contemplated, the patient must sign a release that promises to absolve the clinician of liability for any claims arising from the treatment. ${ }^{22}$

\section{CONCLUSION}

Orthodontists have over the last century enjoyed a comparatively litigation-free era. Unfortunately, this environment has changed and clinicians need to become more aware of the issues in practice that could lead to litigation.

This article has classified the patientorthodontist experience into pre-treatment, active treatment and post-treatment time periods, highlighting the issues pertinent to risk management. The overriding principle remains the need to know and anticipate the risk factors and then practise with the philosophy of prevention.

1. Graber T M, Eliades T, Athanasiou A E. Risk management in orthodontics: experts' guide to malpractice. Chicago: Quintessence Publishing, 2004.

2. Jerold L. The professional ego at work. Am J Orthod Dentofacial Orthop 2006; 130: 555-556.

3. Jerold L. Risk assessment regarding orthodontic communications. In Mizrahi E (ed) Orthodontic pearls. pp 46-50. London: Taylor \& Francis Group, 2004.

4. Ericson S, Kurol J. Early treatment of palatally erupting maxillary canines by extraction of primary canines. Eur J Orthod 1988; 10: 283-295.

5. Ackerman J L, Proffit W R. Diagnosis and treatment planning in orthodontics. In Graber T M, Swain B F (eds) Current orthodontic concepts and techniques. 2nd ed. pp 1-110. Philadelphia: WB Saunders Company, 1975.

6. Graber T M. Diagnosis. In Graber T M (ed) Current orthodontic concepts and techniques. pp 1-55. Philadelphia: WB Saunders Company, 1969.

7. Mizrahi E. Orthodontic investigations. In Mizrahi E (ed) Orthodontic pearls. pp 31-40. London: Taylor \& Francis Group, 2004.

8. Athanasiou A E, Graber T M. Temporomandibular disorders and orthodontics. In Graber T M, Eliades T, Athanasiou A E (eds) Risk management in orthodontics: experts' guide to malpractice. pp 145-164. Chicago: Quintessence Publishing, 2004.

9. Vanarsdall R L. Malpractice aspects of orthodontic treatment in patients with periodontal disease. In Graber T M, Eliades T, Athanasiou A E (eds) Risk management in orthodontics: experts' guide to malpractice. pp 191-206. Chicago: Quintessence Publishing, 2004.

10. Papaconstantinou S. Metabolic profile of orthodontic patients exhibiting root resorption. In Graber T M, Eliades T, Athanasiou A E (eds) Risk management in orthodontics: experts' guide to malpractice. pp 47-73. Chicago: Quintessence Publishing, 2004

11. Vlaskalic V, Boyd R L, Baumrind S. Etiology and sequelae of root resorption. Semin Orthod 1999; 4: 124-131.

12. Levander $E$, Malmgren O. Evaluation of the risk of root resorption during orthodontic treatment: a study of upper incisors. Eur J Orthod 1988; 10: $30-38$.

13. Ericson $\mathrm{S}$, Kurol J. Resorption of incisors after ectopic eruption of maxillary canines: a CT study. Angle Orthod 2000; 70: 415-423.

14. Becker A, Chaushu S. Long-term follow-up of severely resorbed maxillary incisors after resolution of an impacted canine. Am J Orthod Dentofacial Orthop 2005; 127: 650-654.

15. Isaacson K G, Thom A R. Guidelines for the use of 
radiographs in clinical orthodontics. London: British Orthodontic Society, 2001.

16. Smale I, Artun J, Behbehani F et al. Apical root resorption 6 months after initiation of fixed orthodontic appliance therapy. Am J Orthod Dentofacial Orthop 2006: 128: 57-67.

17. Mizrahi E. Case discussion. In Mizrahi E (ed) Orthodontic pearls. pp 41-50. London: Taylor \&t Francis Group, 2004
18. Ernst $S$, Elliot T, Patel A et al. Consent to orthodontic treatment - is it working? Br Dent J 2007; 202: E25.

19. Milton $T$, Hearing $S$, Ireland A J. Ingested foreign bodies associated with orthodontic treatment; report of three cases and review of ingestion/aspiration incident management. Br Dent J 2001; 190: 592-596.

20. Weinberger T. Headgear safety - a simple solution. In Mizrahi E (ed) Orthodontic pearls. pp 106-108. London: Taylor \&t Francis Group, 2004.
21. Jerold L. Integrating the fourth dimension into orthodontic administration. Am J Orthod Dentofacial Orthop 2007; 131: 288-291.

22. Franklin E. Avoiding malpractice lawsuits: lessons learned from actual malpractice case histories. In Graber T M, Eliades T, Athanasiou A E (eds) Risk management in orthodontics: experts' guide to malpractice. pp 207-221. Chicago: Quintessence Publishing, 2004 